\title{
Association between preterm birth and intrauterine growth retardation and child
} asthma

\author{
Bengt Källén*, Orvar Finnström* , Karl-Gösta Nygren ${ }^{\uparrow,+}$ and Petra Otterblad \\ Olausson $^{\S}$
}

ABSTRACT: An association between preterm birth and an increased risk of childhood asthma has been demonstrated, but the importance of intrauterine growth retardation on asthma risk is unclear.

Using data from Swedish health registers, infant characteristics and childhood asthma were studied. Analyses were made using Mantel-Haenszel methodology with adjustment for year of birth, maternal age, parity, smoking in early pregnancy and maternal body mass index. Preterm birth, birth weight and birth weight for gestational week were analysed and childhood asthma was evaluated from prescriptions of anti-asthmatic drugs. Neonatal respiratory problems and treatment for them were studied as mediating factors.

Both short gestational duration and intrauterine growth retardation appeared to be risk factors and seemed to act separately. The largest effect was seen from short gestational duration. Use of mechanical ventilation in the newborn period and bronchopulmonary dysplasia were strong risk factors. A moderately increased risk was also seen in infants born large for gestational age.

We conclude that preterm birth is a stronger risk factor for childhood asthma than intrauterine growth disturbances; however, the latter also affects the risk, and is also seen in infants born at term.

KEYWORDS: Birth weight, epidemiology, mechanical ventilation, registers, respiratory problems

M any publications have investigated the asthma risk in individuals born preterm or with low birth weight (review in [1]). Most studies have investigated short gestational duration or low birth weight, but some refer also to the effect of intrauterine growth retardation (IUGR) [2-5].

Two large recent studies, both from Sweden, have investigated this question and reached different results in spite of similar materials studied $[6,7]$. In the first paper, only extreme preterm birth $(<28$ weeks) was associated with an increased risk of asthma; in the latter study, a continuous decline in asthma risk was seen with increasing gestational duration, statistically significant even at 3738 weeks compared to the reference $39-41$ weeks.

In the present study, we have separated the effect of short gestational duration and low birth weight from IUGR on asthma risk.

\section{MATERIAL AND METHODS}

We used information in the Swedish Medical Birth Register [8] in order to identify children surviving the neonatal period, who were born preterm and/or had a disturbed intrauterine growth. Pregnancy duration as recorded in the register was, in most instances, based on secondtrimester ultrasound or, if absent, on the last menstrual period. Intrauterine growth deviation was expressed as a standard deviation score (SDS) from the normal birth weight at a specific week, estimated according to data in the register [9]. Comparisons were also made with the normal graph published by MARSÁL et al. [10], which gave similar results (not shown). IUGR was defined as $<2$ standard deviations below the normal weight at that gestational week.

This register also gave information on variables that were used in the adjusted analyses: year of delivery, maternal age, parity, smoking habits in early pregnancy, maternal pre-pregnancy body mass index (BMI), maternal country of birth and sex of infant. Information on smoking in early pregnancy and pre-pregnancy BMI was obtained by midwife interviews at the first antenatal visit, usually before the end of the first trimester.

In order to identify asthma in the children, the Swedish Prescribed Drug Register [11] was used. This register covers all prescriptions filled in Sweden since July 1, 2005 and individuals who

\section{AFFILIATIONS}

${ }^{*}$ Tornblad Institute, University of Lund, Lund,

\#Dept of Paediatrics, Linköping University Hospital, Linköping, "IVF and Fertility Clinic, Sophiahemmet,

${ }^{+}$Institute of Medical Epidemiology and Biostatistics, Karolinska Institute, and

${ }^{\S}$ Dept of Statistics, Monitoring and Analyses, National Board of Health and Welfare, Stockholm, Sweden.

CORRESPONDENCE

B. Källén

Tornblad Institute

Biskopsgatan 7

SE-223 62 Lund

Sweden

E-mail: Bengt.Kallen@med.lu.se

Received:

March 082012

Accepted after revision:

June 022012

First published online:

June 142012 
had received prescriptions for anti-asthmatic drugs (Anatomical Therapeutic Chemical code R03) were identified from that date until the end of 2008. In order to increase the probability that the drugs had been prescribed for asthma and not for unrelated complaints, prescriptions made on five or more different occasions were used as a definition of asthma. The age of the children varied between 2 and 11 yrs when asthma was identified.

The association between gestational duration and asthma risk was studied in singletons with known gestational duration and infant sex whose mothers were born in Sweden. The MantelHaenszel technique was used to adjust for year of birth, maternal age, parity, smoking in early pregnancy, maternal pre-pregnancy BMI and infant sex. The adjustment for infant sex was made because the sex ratio declines with gestational week of delivery and there is a male excess in children who are prescribed drugs for asthma. As a reference group, pregnancy duration between 39 and 41 weeks was used. Separate estimates were made for males and females, and analyses were repeated with restriction to infants with a SDS between -1 and $1 \mathrm{SD}$ from the expected weight given pregnancy week of birth, infant sex and parity.

The association between infant birth weight and asthma risk was adjusted in the same way and we used 3,000-3,999 $\mathrm{g}$ as a reference. The analyses were repeated separately for males and females and with restriction to infants with a SDS between -1 and $1 \mathrm{SD}$ from the expected weight given pregnancy week, infant sex and parity.

The association between infant intrauterine growth and asthma risk was adjusted in the same way and we used the SDS -1-1 as a reference. The analyses were made separately for males and females and were repeated with restriction to infants born at 39-41 weeks duration.

The impact of neonatal respiratory diagnoses, mechanical ventilation, continuous positive airway pressure (CPAP) treatment and chorioamnionitis was investigated after adjustment for year of birth, maternal age, parity, smoking in early pregnancy, maternal BMI, infant sex and gestational duration (classified as in table 1). Respiratory diagnoses were defined as specific codes according to the International Classification of Diseases (ICD)-10, P21-P28. P21 defines birth asphyxia, P22 respiratory distress of the newborn, P23 congenital pneumonia, P24 neonatal aspiration syndrome, P25 interstitial emphysema and related conditions, P26 pulmonary haemorrhage, P27 chronic respiratory disease, and P28 other respiratory conditions, all originating in the perinatal period. The presence of chorioamnionitis was identified from the ICD-10 code O41.1.

\section{Ethics}

The study was performed within the responsibilities of the National Board of Health and Welfare (Stockholm, Sweden) and therefore no ethical approval from outside ethical committees was needed.

\section{RESULTS}

The study on gestational duration was made on 765,792 children. Among them, 43,387 (5.7\%) were identified as having filled at least five prescriptions for anti-asthmatic drugs during the study period. The material used for the study of intrauterine growth was slightly smaller (because of some unknown birth weights): the total number of children was 763,666 , among whom 43,184 (5.7\%) had filled at least five prescriptions.

There is a male excess at preterm birth (sex ratio 1.19, 95\% CI 1.17-1.22; the sex ratio among all infants born in weeks 39-41 is 1.03). There is also a male excess among children who had five or more prescriptions for anti-asthmatic drugs (sex ratio 1.67, 95\% CI 1.57-1.79).

Table 1 shows data for the association between gestational duration and the use of anti-asthmatics. The asthma risk decreases with increasing gestational duration at birth and is slightly decreased among post-term births. Similar findings based on birth-weight data are seen in table 2. Risk estimates are however lower than those for gestational duration.

Intrauterine growth changes, estimated as SDSs of birth weight at each gestational duration, also affected the risk for asthma (table 3). The effects on asthma risk were lower than those for gestational duration.

As short gestational duration, birth weight and IUGR are associated, the analysis of the effect of gestational duration or birth weight on asthma risk was repeated with a restriction to children who had not deviated markedly in intrauterine growth at birth (SDS -1-1) (tables 1 and 2). The estimated odds ratios were relatively similar to those obtained without restrictions based on intrauterine growth, but confidence intervals were wider.

Similarly, the effect of disturbed intrauterine growth (estimated as SDS of birth weight from expected weight at that gestational week) was analysed, restricted to children who were born in weeks 39-41 (table 3). When these data were compared with those obtained without restrictions to pregnancy duration, no marked differences appeared.

In order to study possible confounding from maternal asthma, females who had reported the use of anti-asthmatic drugs in early pregnancy were removed from the analysis $(n=13,897)$. Only small changes in the estimated odds ratios were seen (data not shown).

The various analyses were run separately for males and females but no consistent difference between sexes in asthma risk associated with gestational duration or intrauterine growth was noted (data not shown).

Table 4 presents the results of the effect of respiratory diagnosis, mechanical ventilation and CPAP on asthma risk. The highest risk estimate was found after bronchopulmonary dysplasia. The risk was also high after mechanical ventilation. Also, in the absence of a recorded mechanical ventilation or CPAP procedure, an increased asthma risk was found.

The effect of chorioamnionitis did not reach statistical significance. There were 98 infants with such a maternal diagnosis and childhood asthma, and 982 among all infants born. The adjusted odds ratio was 1.25 (95\% CI 0.97-1.61). 
TABLE 1 Number of children in population and number with asthma according to gestational week at birth

\begin{tabular}{|c|c|c|c|c|c|c|}
\hline \multirow{2}{*}{$\begin{array}{l}\text { Gestational age } \\
\text { weeks }\end{array}$} & \multicolumn{3}{|c|}{ All children } & \multicolumn{3}{|c|}{ Children with SDS -1-1 } \\
\hline & Population & With asthma & OR $(95 \% \mathrm{Cl})$ & Population & With asthma & OR $(95 \% \mathrm{Cl})$ \\
\hline $23-27$ & 1570 & 280 & $4.06(3.59-4.59)$ & 650 & 121 & $4.17(3.45-5.05)$ \\
\hline $28-30$ & 2454 & 329 & $2.87(2.56-3.22)$ & 1045 & 156 & $3.25(2.75-3.83)$ \\
\hline $31-32$ & 3157 & 363 & $2.28(2.05-2.54)$ & 1602 & 174 & $2.22(1.90-2.59)$ \\
\hline $37-38$ & 141683 & 9323 & $1.43(1.39-1.47)$ & 90190 & 5724 & $1.38(1.34-1.43)$ \\
\hline $39-41$ & 528793 & 27439 & 1.00 (reference) & 342941 & 17771 & 1.00 (reference) \\
\hline $42-44$ & 56885 & 2864 & $0.89(0.86-0.93)$ & 35545 & 1757 & $0.90(0.85-0.94)$ \\
\hline Total & 765792 & 43387 & & 490683 & 27368 & \\
\hline
\end{tabular}

Data are presented as $\mathrm{n}$, unless otherwise stated. Data were adjusted for year of birth, maternal age, parity, smoking in early pregnancy, pre-pregnancy body mass index and sex of infant. SDS: standard deviation score.

\section{DISCUSSION}

Our results indicated that both preterm birth and IUGR are risk factors for childhood asthma. The strongest effects were seen in preterm birth and also in infants who showed no marked deviation in intrauterine growth. A weaker effect was seen in small-for-date infants, also when born at term. Also infants who were large for gestational age seemed to show a moderately increased asthma risk.

The present study is large and it has been possible to adjust for a number of putative confounders. The identification of asthma in the children relied on prescription of anti-asthmatic drugs. These may be used for other medical problems than asthma, which would dilute the material with non-asthma cases. By restricting the definition to the filling of at least five prescriptions during the observation period, the proportion of true asthma cases should reasonably increase but some non-asthma cases may still have been included in the asthma group. This will result in a bias of the odds ratio estimates towards 1.0, if there is no effect on the non-asthma cases. Many studies have used prescription of antiasthmatic drugs for the definition of asthma in register studies [6, $7,12,13]$. One study compared the physician diagnosis of asthma with the prescription of anti-asthmatic drugs and found slightly more potential asthmatics based on prescription data than on physician diagnosis, but the sensitivity was $91 \%$ and the specificity $98 \%$ [14].

Most studies on premature birth and asthma in the offspring have indicated an association even though the risk estimates have varied [1]. Less attention has been paid to the possible effect of intrauterine growth disturbances for the origin of child asthma. Three studies $[2,4,15]$ found no clear effect of IUGR, but in a twin study it was demonstrated that IUGR increased the asthma risk [5], and in a study of prematurely born infants, the presence of IUGR increased the risk for asthma [3]. In a recent study [6], adjustment for intrauterine fetal growth removed the effect of short gestational age on asthma risk, except for the shortest stratum, 23-27 weeks gestation.

TABLE 2 Number of children in population and number with asthma according to birth weight

\begin{tabular}{|c|c|c|c|c|c|c|}
\hline \multirow[t]{2}{*}{ Birth weight $\mathbf{g}$} & \multicolumn{3}{|c|}{ All children } & \multicolumn{3}{|c|}{ Children with SDS -1-1 } \\
\hline & Population & With asthma & OR (95\% Cl) & Population & With asthma & OR (95\% Cl) \\
\hline$<1000$ & 1758 & 269 & $3.23(2.85-3.66)$ & 502 & 86 & $3.78(3.02-4.73)$ \\
\hline $1000-1499$ & 2540 & 333 & $2.68(2.39-3.00)$ & 910 & 142 & $3.55(2.48-4.23)$ \\
\hline $1500-2499$ & 19046 & 1783 & $1.91(1.81-2.01)$ & 6254 & 670 & $2.20(2.03-2.38)$ \\
\hline 4000-4999 & 156838 & 8765 & $0.94(0.92-0.97)$ & 35564 & 1961 & $0.94(0.92-0.97)$ \\
\hline $5000-6499$ & 4485 & 251 & $0.88(0.77-1.00)$ & & & \\
\hline$\geqslant 6500$ & 5 & 0 & & & & \\
\hline Total & 764209 & 43264 & & 496712 & 27325 & \\
\hline
\end{tabular}

Data are presented as n, unless otherwise stated. Data were adjusted for year of birth, maternal age, parity, smoking in early pregnancy, pre-pregnancy body mass index and sex of infant. SDS: standard deviation score. 


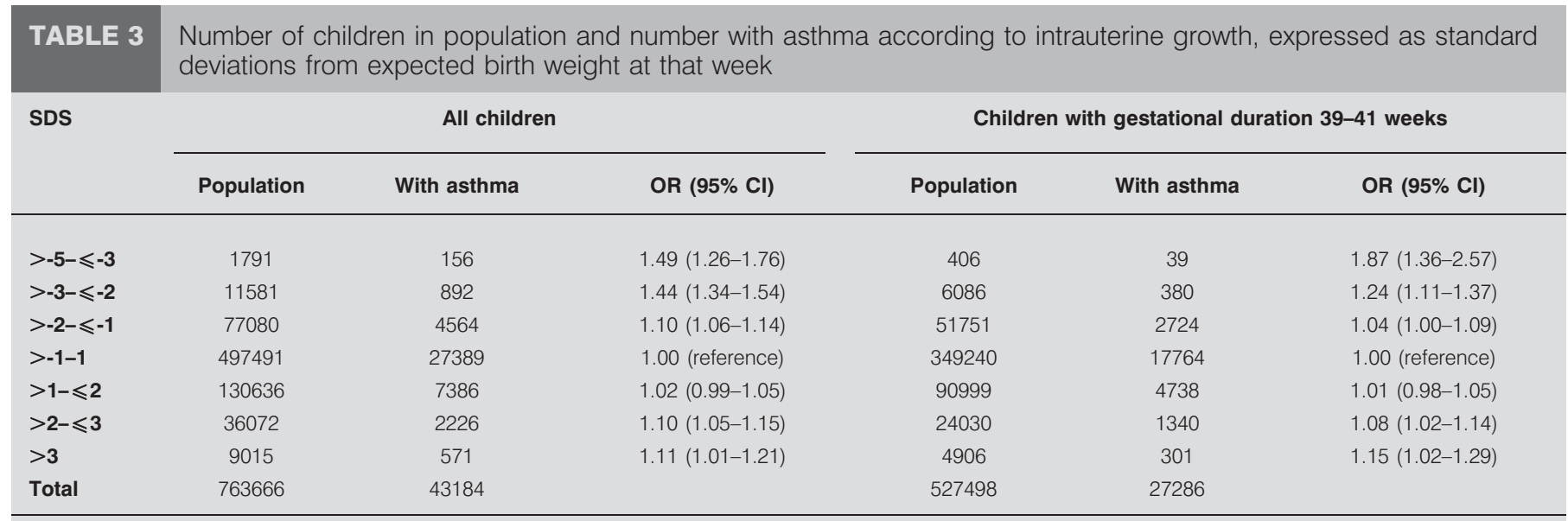

Data are presented as n, unless otherwise stated. Data were adjusted for year of birth, maternal age, parity, smoking in early pregnancy, pre-pregnancy body mass index and sex of infant. SDS: standard deviation score.

There is a strong interaction between short gestational duration and IUGR: fetuses with impaired intrauterine growth have a tendency to be born preterm, either iatrogenically or because of placental insufficiency. It is therefore difficult to study the two phenomena independently. In the present study, both short gestational duration and IUGR were associated with an increased asthma risk. In order to separate the two variables, the effect of gestational duration was studied in children who had shown no signs of major intrauterine growth deviation (from -1 to 1 standard deviation from expected weight) and the effect of intrauterine growth deviation in term children, born at 39-41 weeks gestation. Children who had shown no major deviation in intrauterine growth but were born after short gestational duration or with low birth weight had a higher asthma risk than children who were born term with signs of IUGR. Both factors thus seem to be of importance even though short gestational duration appears to be the dominating one.

\begin{tabular}{|c|c|c|c|c|}
\hline TABLE 4 & \multicolumn{4}{|c|}{$\begin{array}{l}\text { Risk for asthma in children with respiratory } \\
\text { problems in the neonatal period }\end{array}$} \\
\hline \multicolumn{2}{|c|}{ Group of children } & $\begin{array}{l}\text { With } \\
\text { asthma }\end{array}$ & $\begin{array}{c}\text { Total } \\
\text { number }\end{array}$ & OR $(95 \% \mathrm{Cl})$ \\
\hline \multicolumn{5}{|c|}{ Respiratory diagnosis } \\
\hline \multicolumn{2}{|c|}{ Mechanical ventilation or CPAP } & 2762 & 29827 & $1.33(1.26-1.39)$ \\
\hline \multicolumn{2}{|c|}{ No mechanical ventilation or CPAP } & 2276 & 25921 & $1.32(1.26-1.39)$ \\
\hline \multicolumn{2}{|c|}{ CPAP } & 419 & 3508 & $1.22(1.07-1.39)$ \\
\hline \multicolumn{2}{|c|}{ Mechanical ventilation } & 126 & 710 & $1.85(1.44-2.31)$ \\
\hline \multicolumn{2}{|c|}{ Bronchopulmonary dysplasia } & 39 & 176 & $1.97(1.12-3.47)$ \\
\hline
\end{tabular}

Data are presented as $n$, unless otherwise stated. Data are shown after adjustment for year of birth, maternal age, parity, smoking in early pregnancy, maternal body mass index and gestational duration (groups as in tables 1 and 3). Children with asthma (five or more prescriptions) $n=43,387$, total children $n=765,792$. CPAP: continuous positive airway pressure.
Post-term birth or high birth weight was associated with a decrease in asthma risk but children born large for gestational age showed a moderately increased risk. In a previous study, large birth weight was associated with a decreased asthma risk [16] but post-term or large-for-date infants were not studied. That paper also found a possibly increased risk at extremely high birth weights $(>6.5 \mathrm{~kg})$ but statistical significance was not reached. Only a few such infants were included in the present study.

Various explanations of the association between preterm birth and an increased risk for asthma have been discussed [1] and there are probably complex causal pathways. The risk increase is probably not due to atopy, which is reduced in incidence after preterm birth [17]. One possible model [1] is a direct effect of preterm birth per se on asthma risk, regardless of obstetric risk factors such as chorioamnionitis [18] or post-natal complications as respiratory diseases and post-natal asphyxia, as discussed below. Premature birth per se leads to both transient and persistent changes in lung development [19], which can make the lungs more susceptible to later asthmacausing exposures. The post-natal growth rate was also of importance in an animal model [20].

Another model [1] implies that genetics or conditions during pregnancy resulted in both preterm birth and an increased risk for asthma. Parental asthma increases the child's risk of asthma due to genetics and, as maternal asthma is associated with an increased risk for preterm birth [21], maternal asthma could act as a confounder. Exclusion of females who had reported the use of anti-asthmatic drugs in early pregnancy hardly affected the risk estimate for child asthma, however. Some maternal factors like smoking are associated with a moderately increased risk for preterm birth and may be causal in the origin of child asthma [15]. We therefore adjusted for maternal smoking and we did the same for maternal age, parity and BMI.

The main reason for our study was not to identify the relevant mechanisms but to try to separate the effect of preterm birth 
and IUGR. Neonatal respiratory problems are common in infants born preterm and we found that the presence of a neonatal respiratory diagnosis increased the asthma risk after adjustment for gestational duration, notably when mechanical ventilation had been needed and/or bronchopulmonary dysplasia was present. Mechanical ventilation has previously been shown to be a risk factor for asthma among infants with a birth weight $<1,500 \mathrm{~g}$ [22]. Whether the effect we found was due to the procedure or the occurrence of more severe respiratory problems could not be concluded. It is, however, noticeable that the use of CPAP did not further increase the asthma risk.

As the development of the alveoli and the small airways occurs late in fetal life and post-natally, preterm birth and neonatal factors associated with it may result in lung damage that, later in life, increases the risk for asthma [23, 24]. Experimentally, even a short period of mechanical ventilation resulted in a persistent bronchiolar remodelling, which may increase the risk for future asthma development [25]. The significance of bronchopulmonary dysplasia for future asthma risk has also been stressed [26].

An increased asthma risk has been described in children who were exposed to chorioamnionitis [18]. We found a moderately increased risk but statistical significance was not reached.

IUGR also seems to be a risk factor for asthma among term infants. Various possible explanations for such an association have been discussed. Normal lung development depends on the presence of appropriate oxygen tension and nutrition and, thus, lung anomalies may occur in individuals with IUGR that increase the risk of asthma [27]. Using magnetic resonance imaging, reduced lung volume was found in fetuses that had intrauterine growth restrictions [28]. In experiments with rats, an increase in inflammatory and profibrotic processes was found after protein restriction during gestation, leading to IUGR, and thus affected future lung function [29].

In conclusion, our analysis suggests that even though IUGR is associated with an increased risk of asthma, short gestational duration even in the absence of marked IUGR is a stronger risk factor. The immaturity of the lungs in infants born preterm and/or the necessary treatment may cause permanent damage which increases the risk for childhood asthma.

\section{STATEMENT OF INTEREST}

None declared.

\section{REFERENCES}

1 Jaakkola JJK, Ahmed P, Ieromnimon A, et al. Preterm delivery and asthma: a systematic review and meta-analysis. J Allergy Clin Immunol 2006; 118: 823-830.

2 Jaakola JJK, Gissler M. Maternal smoking in pregnancy, fetal development, and childhood asthma. Am J Publ Health 2004; 94 136-140.

3 Greenough A, Yuksei B, Cheeseman P. Effect of in utero growth retardation on lung function at follow-up of prematurely born infants. Eur Respir J 2004; 24: 731-733.
4 Gessner BD, Chimonas M-A. Asthma is associated with preterm birth but not with small for gestational age status among a population-based cohort of Medicaid-enrolled children $<10$ years of age. Thorax 2007; 62: 231-236.

5 Örtqvist AK, Lundholm C, Carlström E, et al. Familial factors do not confound the association between birth weight and childhood asthma. Pediatrics 2009; 124: e737-e743.

6 Crump C, Winkleby MA, Sundquist J, et al. Risk of asthma in young adults who were born preterm: a Swedish national cohort study. Pediatrics 2011; 127: e913-e920

7 Vogt H, Lindström K, Bråbäck L, et al. Preterm birth and inhaled corticosteroid use in 6- to 19-year-olds: a Swedish national cohort study. Pediatrics 2011; 127: 1052-1059.

8 National Board of Health and Welfare Centre for Epidemiology. The Swedish Medical Birth Register - a Summary of Content and Quality. 2003. www.sos.se/publikationer2003/2003-112-3 Date last accessed: February 2, 2012.

9 Källén B. A birth weight for gestational age standard based on data in the Swedish Medical Birth Registry 1985-1989. Eur J Epdemiol 1995; 11: 601-606.

10 Marsál K, Person PH, Larsen T, et al. Intrauterine growth curves based on ultrasonically estimated foetal weights. Acta Paediatr 1996; 85: 843-848.

11 Wettermark B, Hammar N, Fored CM, et al. The new Swedish Prescribed Drug Register - opportunities for pharmacoepidemiological research and experience from the first six months. Pharmacoepidemiol Drug Safety 2007; 16: 726-735.

12 Yuan W, Fonager K, Olsen J, et al. Prenatal factors and use of antiasthma medications in early childhood: a population-based Danish birth cohort study. Eur J Epidemiol 2003; 18: 763-768.

13 Metsälä J, Kilkkinen A, Kalla M, et al. Perinatal factors and the risk of asthma in childhood - a population-based register study in Finland. Am J Epidemiol 2008; 168: 170-178.

14 Bianchi M, Clavenna A, Sequi $\mathrm{M}$, et al. Asthma diagnosis vs. analysis of anti-asthmatic prescriptions to identify asthma in children. Eur J Clin Pharmacol 2011; 67: 967-968.

15 Jaakola JJK, Gissler M. Are girls more susceptible to the effects of prenatal exposure to tobacco smoke on asthma? Epidemiology 2007; 18: $573-576$

16 To T, Guan J, Wang C, et al. Is large birth weight associated with asthma risk in early childhood? Arch Dis Child 2010; 97: 169-171.

17 Siltanen M, Wehkalampi K, Hovi $\mathrm{P}$, et al. Preterm birth reduces the incidence of atopy in adulthood. J Allergy Clin Immunol 2011; 127: 935-942.

18 Getohun D, Strickland D, Zeiger RS, et al. Effect of chorioamnionitis on early childhood asthma. Arch Pediatr Adolesc Med 2010; 164: 187-192.

19 Cock M, Hanna M, Sozo F, et al. Pulmonary function and structure following mild preterm birth in lambs. Pediatr Pulmonol 2005; 40: 336-348.

20 Snibson K, Harding R. Postnatal growth rate, but not mild preterm birth, influences airway structure in adult sheep challenged with house mite. Exp Lung Res 2008; 34: 69-84.

21 Källén B, Otterblad Olausson P. Use of anti-asthmatic drugs during pregnancy. 2. Infant characteristics excluding congenital malformations. Eur J Clin Pharmacol 2007; 63: 375-381.

22 Mai XM, Gäddlin PO, Nilsson L, et al. Asthma lung function and allergy in 12-year-old children with very low birth weight: a prospective study. Pediatr Allergy Immunol 2003; 14: 184-192.

23 Maritz GS, Morley CJ, Harding R. Early developmental origins of impaired lung structure and function. Early Human Develop 2005 81: 763-771

24 Harding R, Maritz G. Maternal and fetal origins of lung disease in adulthood. Seminars Fetal Neonatal Med 2012; 17: 67-72. 
25 O'Reilly M, Hooper SB, Allison BJ, et al. Persistent bronchiolar remodeling following brief ventilation of the very immature ovine lung. Am J Physiol Lung Cell Mol Physiol 2009; 297: L992-L1001.

26 Baraldi E, Filippone M. Chronic lung disease after premature birth. N Engl J Med 2007; 357: 1946-1955.

27 Pike K, Pillow J, Lucas JS. Long term respiratory consequences of intrauterine growth restriction. Semin Fetal Neonatal Med 2012; 12: 92-98.
28 Darmodaram MS, Story L, Eixarch E, et al. Foetal volumetry using magnetic resonance imaging in intrauterine growth restriction. Early Hum Dev 2012; 88: Suppl. 1, S35-S40.

29 Alejandre Alcazar A, Ostreicher I, Appel S, et al. Developmental regulation of inflammatory cytokine-mediated Stat3 signalling: the missing link between intrauterine growth restriction and pulmonary dysfunction? J Mol Med 2012; 90: 945-957. 